derungen bei der Mukosa-Architektur im Duodenum nachweisebar waren, kann laut Autoren zwei Gründe haben: Entweder der Zellschaden bei NCGS ist nicht im Duodenum, sondern in tieferen Darmabschnitten lokalisiert oder er zeigt sich eher subklinisch.

Die gefundenen Marker stehen wohl tatsächlich in Zusammenhang mit dem Glutenverzehr: Nach sechs Monaten glutenfreier Ernährung gaben alle 20 NCGS-Betroffenen deutlich weniger Symptome an und zeigten einen signifikanten Abfall der zuvor identifizierten Marker. Jedoch korrelierte das Ausmaß der gemessenen Marker nicht signifikant mit dem der Symptomreduktion. Wie die Ernährung der 20 Betroffenen während der Diätzeit konkret aussah, geht nicht hervor. Folglich kann nur vermutet werden, dass der Glutenverzicht in kausalem Zusammenhang mit den beobachteten Verbesserungen steht. Andere Inhaltsstoffe glutenhaltiger Nahrungsmittel oder eine Ernährungsweise, die sich günstig auf die Darmbarriere auswirkt, könnten die Beobachtungen ebenso erklären.

Fazit: Die Ergebnisse weisen auf das Vorhandensein objektiver Marker einer systemischen Immunaktivierung und eines intestinalen Epithelzellschadens hin, die zukünftig bei der Identifizierung von NCGS-Betroffenen eine Rolle spielen könnten. Die Autoren betonen aber, dass es in der Studie nicht darum ging, den potenziellen Pathomechanismus oder die verantwortlichen Trigger zu eruieren, die zur Darmbarrierestörung führen. Dies müsse, ebenso wie die Eignung der identifizierten Marker im Rahmen der Diagnostik und Therapie, durch weitere Studien geklärt werden.

Dr. Imke Reese

Uhde M et al., Intestinal cell damage and systemic immune activation in individuals reporting sensitivity to wheat in the absence of coeliac disease. Gut 2016; doi: 10.1136/gutjnl-2016-311964

\title{
Allergie gegen Krusten- und Schalentiere: Vorsicht bei der Diagnostik
}

\section{Allergische Reaktionen auf Krusten- oder Schalentiere können lebensbedrohlich verlaufen. Bei Verdacht auf eine schwere Allergie sollte daher vor jedem In-vivo-Test allergenspezifisches IgE bestimmt werden.}

samt-IgE, allergenspezifisches IgE mit Blick auf die auslösende Krusten- oder Schalentier-Spezies und allergenspezifisches IgE gegen TM (Pen a 1), das derzeit einzige erhältliche TM für eine gezielte (Singleplex-)Testung.

Fallen Extrakt- und TM-spezifische IgE-Ergebnisse positiv aus und ist der Spiegel von TM-spezifischem IgE höher als der für den Gesamtextrakt, ist eine immundominante Sensibilisierung gegen Krustentier-TM wahrscheinlich. Es ist dann mit einer breiten serologischen Kreuzreaktivität auf andere Krustenund Schalentierspezies zu rechnen. Die Symptome müssen aber zur identifizierten Tierspezies passen. Nur wenn Symptome und Testergebnis korrespondieren, ist eine klinisch relevante Allergie nachgewiesen. Ist nur der Test auf Extrakt-, nicht aber auf TM-spezifisches IgE positiv, ist eine Sensibilisierung auf TM unwahrscheinlich. Jedoch könnten andere Allergene eine Rolle spielen.

Ein Hauttest ist unumgänglich, wenn beide IgE-Tests negativ sind. Verwendet wird entweder ein kommerzieller Extrakt oder Nativmaterial. Ein klar posi- tiver Hauttest lässt auf eine Sensibilisierung vom Soforttyp schließen. Ist der Hauttest hingegen ebenfalls negativ, macht dies eine IgE-vermittelte Sensibilisierung unwahrscheinlich.

Orale Provokationstests sind Fällen vorbehalten, in denen Anamnese und diagnostische Resultate nicht übereinstimmen. Ein negatives Ergebnis schließt eine Allergie auf Krusten- und Schalentiere in der Regel aus.

Dr. Robert Bublak

Lopata AL et al. Allergens and Molecular Diagnostics of Shellfish Allergy. Allergo J Int 2016;25:216-24

\section{Kommentar}

Die Komplexität der Diagnostik einer Allergie gegen Krusten und Schalentiere wird vermutlich von den meisten Allergologen unterschätzt. Die aktuelle Übersichtsarbeit von Prof. Lopata, Townsville, Australien beschreibt umfassend die bisher bekannten Allergene und Kreuzreaktivitäten sowie den potentiellen Nutzen der molekularen Allergiediagnostik. Abgerundet wird der Beitrag durch klare Empfehlungen zum diagnostischen Vorgehen im klinischen Alltag, sodass sich die Lektüre des Beitrages im Orginal auf jeden Fall lohnt.

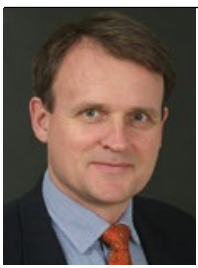

Prof. Dr. Thilo Jakob Direktor der Klinik für Dermatologie und Allergologie Universitätsklinikum Gießen u. Marburg, Standort Gießen 